\title{
Temperature-Dependent Characteristics and Single-Mode Performance of AlGaInP-Based 670-690-nm Vertical-Cavity Surface-Emitting Lasers
}

\author{
M. Hagerott Crawford, R. P. Schneider, Jr., K. D. Choquette, and K. L. Lear, Member, IEEE
}

\begin{abstract}
We report on temperature dependent characteristics and single mode performance of one-wave cavity, planar implanted, AlGaInP-based vertical-cavity surface emitting lasers. By optimizing the overlap between the gain peak and the cavity mode of the structure, we demonstrate record device performance, including $8.2 \mathrm{~mW}$ maximum output power and $11 \%$ power conversion efficiency for multimode operation and $1.9 \mathrm{~mW}$ and $9.6 \%$ power conversion efficiency for single mode operation at $687 \mathrm{~nm}$. Improved performance at elevated temperatures is also achieved, with $1.5 \mathrm{~mW}$ output power demonstrated at $50{ }^{\circ} \mathrm{C}$ from a $15-\mu \mathrm{m}$-diameter device.
\end{abstract}

\section{INTRODUCTION}

A DVANCES in growth techniques and heterostructure design of AlGalnP-based materials have led to the recent development of vertical cavity surface emitting lasers (VCSEL's) emitting in the 660-680 $\mathrm{nm}$ range [1]-[3]. In the past year, significant advances in device output power and efficiency have been achieved [4] with the development and optimization of a one-wave cavity design which employs carbon as the sole p-type dopant in the structure [5]. The utility of these red VCSEL's will be gauged by how well they can meet the performance specifications of several technologies for which they are potentially well suited, including laser printing and scanning, plastic fiber-based communications and optical data storage. While each application has distinct device specifications, critical parameters include maximum multimode and single-mode output power, device efficiency and performance at elevated temperatures. In addition, several applications have specific wavelength requirements, such as achieving $650 \mathrm{~nm}$ range emission for relatively low signal attenuation in plastic fiber-based applications. In this letter, we report on progress that has been made in these critical areas, with an emphasis on the wavelength and temperature dependence of the VCSEL performance.

The VCSEL structures described in this paper were grown by MOVPE on (311) GaAs substrates without wafer rotation as described previously [6]. The one-wave optical cavity contains four or five $60 \AA$ compressively strained $\operatorname{In}_{0.56} \mathrm{Ga}_{0.44} \mathrm{P}$

Manuscript received March 8, 1995. This letter was supported by the US Department of Energy under contract no. DE-AC04-94AL85000.

The authors are with the Sandia National Laboratories, Albuquerque, NM 87185-0603 USA.

IEEE Log Number 9412169. quantum wells with $60 \AA\left(\mathrm{Al}_{0.5} \mathrm{Ga}_{0.5}\right)_{0.5} \mathrm{In}_{0.5} \mathrm{P}$ barriers and $\left(\mathrm{Al}_{0.5} \mathrm{Ga}_{0.5}\right)_{0.5} \mathrm{In}_{0.5} \mathrm{P}$ cladding layers on each side of the quantum-well region. The distributed Bragg reflectors (DBR's) are composed of $\mathrm{Al}_{0.5} \mathrm{Ga}_{0.5} \mathrm{As}$ and $\mathrm{AlAs}$ quarter-wave layers, with bi-parabolic grading between the layers to reduce series resistance in the devices [6]. The bottom DBR has 55.5 $\mathrm{Al}_{0.5} \mathrm{Ga}_{0.5} \mathrm{As}-\mathrm{AlAs}$ layer pairs and is doped $\mathrm{n}$-type with silicon to a level of approximately $2 \times 10^{18} \mathrm{~cm}^{-3}$. The top DBR has 34 pairs and is doped with carbon to a similar level. The active region is nominally undoped. Planar gain guided lasers were fabricated in a top emitting geometry with proton implantation to channel current into the active region. Implant defined device diameters ranged from $5-50 \mu \mathrm{m}$.

The fact that the VCSEL wafer was not rotated during growth allows for a range of lasing wavelengths across the wafer and a variation of the spectral overlap between the gain peak and the cavity mode resonance of the laser structure [7]. We take advantage of this variation to explore the optimum conditions for peak output power, minimum threshold current, and improved performance at elevated temperatures. The first wafer that will be discussed contains five quantum wells and was designed with the cavity mode at $673 \mathrm{~nm}$ at the center of the two inch wafer. The spectral position of the cavity mode increases in wavelength approximately linearly toward the upstream edge of the wafer, and the total wavelength variation of the cavity mode from the center to the edge of the wafer is approximately $30 \mathrm{~nm}$. Room temperature photoluminescence measurements of a wafer containing only the quantum-well active region demonstrates a peak at $660 \mathrm{~nm}$ at the center of the wafer. The spectral position of the photoluminescence peak increases by approximately $4 \mathrm{~nm}$ from the center to the upstream edge of the wafer, primarily due to a variation in the In incorporation and, therefore, the strain in the quantum wells.

In Fig. 1, we show the threshold current and peak output power of various $30-\mu \mathrm{m}$ diameter devices at different positions across the wafer. The $x$-axis describes the emission wavelength at subthreshold current so that heating is negligible and therefore corresponds to the spectral position of the cavity mode. We see that the minimum threshold current, and therefore, optimal gain peak/cavity mode overlap [8] occurs for an emission wavelength of approximately $682 \mathrm{~nm}$. The peak output power is seen at an emission wavelength of 


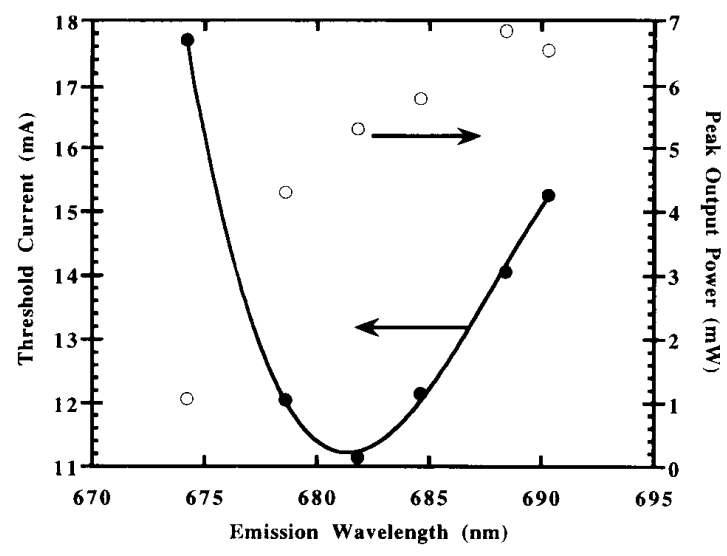

Fig. 1. Threshold current and peak output power of $30-\mu \mathrm{m}-$ diameter VCSEL's at various locations across a two inch wafer. The $x$-axis corresponds to the peak emission wavelength at subthreshold current injection, such that heating is negligible.

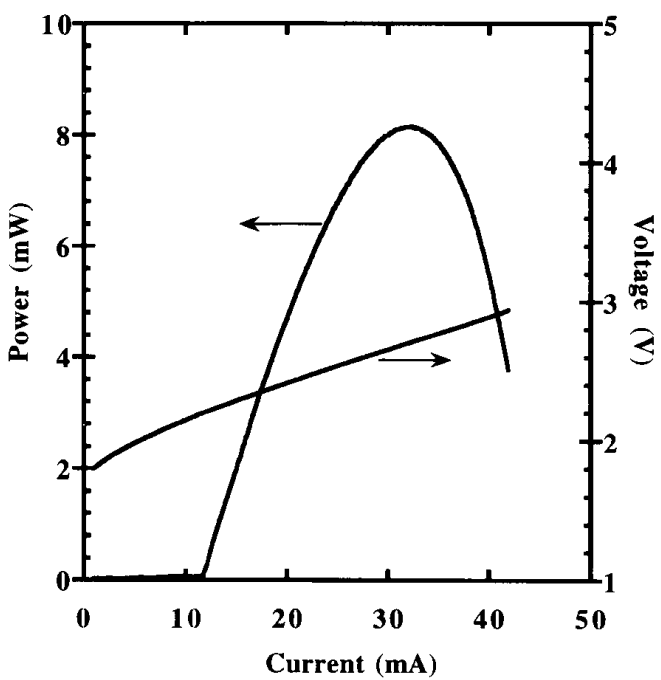

Fig. 2. Light output power-current and current-voltage data for a $30-\mu \mathrm{m}$ diameter VCSEL with emission at $687 \mathrm{~nm}$.

approximately $688 \mathrm{~nm}$, which corresponds to a wafer region where the gain peak is initially blue-shifted with respect to the cavity mode, and can come into alignment with the cavity mode as the device heats [9]. In Fig. 2, we show record performance from a $30-\mu \mathrm{m}$ device emitting at $687 \mathrm{~nm}$. For this device, we achieved $8.2 \mathrm{~mW}$ of multimode output power and $11 \%$ peak power conversion (wallplug) efficiency, where the threshold current (voltage) is $11.7 \mathrm{~mA}(2.2 \mathrm{~V})$.

Intentionally designing the structure with a blue-shifted gain peak enables the gain peak/cavity mode overlap to occur at elevated temperatures and can enable a more constant threshold current over a wider temperature range [8], [9]. In Fig. 3, we show the threshold current for $20-\mu \mathrm{m}$ diameter devices at various positions on the wafer as a function of heatsink temperature. The measurements are performed under continuous current injection conditions and

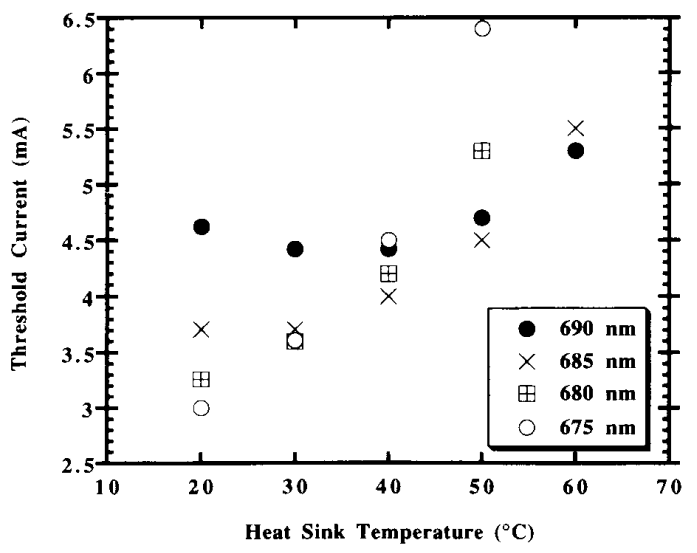

Fig. 3. Threshold current as a function of heatsink temperature for various $20-\mu \mathrm{m}$ diameter VCSEL's across the wafer. The wavelength designation refers to the VCSEL emission wavelength at subthreshold current.

the device substrate has not been thinned or bonded to the heatsink. The threshold current increases with temperature for the shorter wavelength lasers, increasing more dramatically for the shortest wavelengths. In contrast, the threshold current initially decreases with temperature and then increases for the $690 \mathrm{~nm}$ lasers, demonstrating an optimal gain peak/cavity mode overlap at a heatsink temperature of $30^{\circ} \mathrm{C}$. As a result, the threshold current of the $690 \mathrm{~nm}$ lasers is relatively constant over the temperature range of $20-50{ }^{\circ} \mathrm{C}$, varying between $4.4 \mathrm{~mA}$ and $4.8 \mathrm{~mA}$.

A further benefit of an optimal gain peak/cavity mode overlap at elevated temperatures is an extension of the temperature range over which lasing can be obtained. In Fig. 4, we show the L-I characteristics of $15-\mu \mathrm{m}$ devices at two different positions on the wafer for various heatsink temperatures. Fig. 4(a) shows data for a laser from a region of the wafer that corresponds with a cavity mode spectral position of $675 \mathrm{~nm}$. The laser has a relatively low threshold current of $3.4 \mathrm{~mA}$ at $20{ }^{\circ} \mathrm{C}$, but the threshold current increases significantly with increasing temperature, and the output power drops approximately linearly with a slope of $-0.088 \mathrm{~mW} /{ }^{\circ} \mathrm{C}$. In contrast, a laser from the 690 -nm region of the wafer has a much smaller variation in threshold current and a slower power drop of $-0.075 \mathrm{~mW} /{ }^{\circ} \mathrm{C}$, as shown in Fig. 4(b). We see that a peak output power of $1.5 \mathrm{~mW}$ and $0.9 \mathrm{~mW}$ is achieved at $50{ }^{\circ} \mathrm{C}$ and $60^{\circ} \mathrm{C}$, respectively, from the $690-\mathrm{nm}$ device, representing the highest powers achieved to date at these operating temperatures.

Smaller devices were also evaluated to determine optimum conditions for single-mode output. Highest powers were obtained from $10-\mu \mathrm{m}$ diameter devices emitting at $687 \mathrm{~nm}$ from a second wafer containing a four quantum-well active region. The L-I characteristic of a $10-\mu \mathrm{m}$ device is shown in Fig. 5, with the mode spectrum at two drive currents included as an inset. We see from the spectra that the transition from single transverse mode to multiple transverse mode occurs between $6.5 \mathrm{~mA}$ and $7 \mathrm{~mA}$ which corresponds to a single mode output power of greater than $1.9 \mathrm{~mW}$. The device demonstrates a peak wallplug efficiency of $9.6 \%$ at $5.6 \mathrm{~mA}$ and a threshold current 


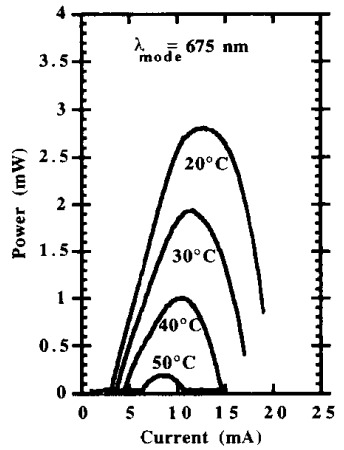

(a)

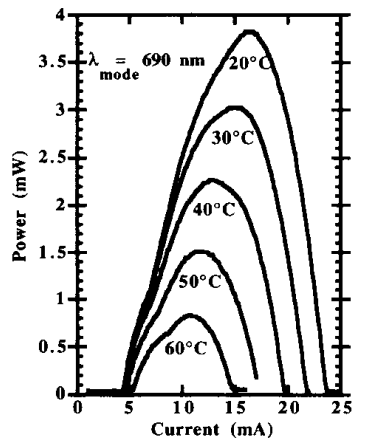

(b)
Fig. 4. Light output power-current data as a function of heatsink temperature for $15-\mu \mathrm{m}$ diameter VCSEL's at wafer locations corresponding to the cavity mode at (a) $675 \mathrm{~nm}$, and (b) $690 \mathrm{~nm}$.

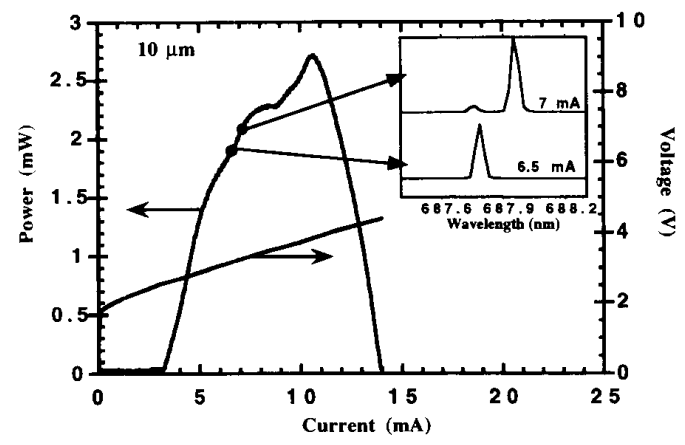

Fig. 5. Light output power-current and current-voltage data for a $10-\mu \mathrm{m}$ diameter VCSEL with emission at $687 \mathrm{~nm}$. The inset shows the emission spectrum at $6.5 \mathrm{~mA}$ and $7 \mathrm{~mA}$ injected current.

of $2.3 \mathrm{~mA}$. While we expect that the single-mode operation of these devices is far from optimized, the powers reported here are already relevant for many fiber-optic applications.

\section{CONCLUSION}

We have evaluated the performance of planar implanted AlGaInP-based 670-690 nm VCSEL's with varying gain peak/cavity mode overlap. Record performance, including $8.2 \mathrm{~mW}$ multimode output power with $11 \%$ peak wallplug efficiency as well as $1.9 \mathrm{~mW}$ single-mode output power with $9.6 \%$ peak wallplug efficiency, has been achieved from VCSEL's emitting at $687 \mathrm{~nm}$. Significant improvements have been achieved in device performance at elevated temperature, with $1.5 \mathrm{~mW}$ output power demonstrated at $50{ }^{\circ} \mathrm{C}$. These advances address a number of device specifications which are critical for the successful implementation of AlGaInP-based visible VCSEL's into optoelectronic applications. Further studies are in progress to establish device reliability as well as to enable operation at shorter wavelengths $(\lambda \leq 650 \mathrm{~nm})$.

\section{ACKNOWLEDGMENT}

The authors acknowledge fruitful discussions with W. W. Chow and G. R. Hadley, as well as the technical assistance of K. M. Geib, J. J. Figiel, S. P. Kilcoyne, and J. A. Nevers.

\section{REFERENCES}

[1] J. A. Lott, R. P. Schneider Jr., K. D. Choquette, S. P. Kilcoyne and J. J. Figiel, "Room temperature continuous wave operation of red vertical cavity surface emitting laser diodes," Electron. Lett., vol. 29 , pp. 1693-1694, 1993.

[2] K. F. Huang, K. Tai, C. C. Wu, and J. D. Wynn, "Continuous-wave visible InGaP/InGaAIP quantum well surface-emitting laser diodes," Electron. Lett., vol. 29, p. 1314, 1993.

[3] R. P. Schneider, Jr., K. D. Choquette, J. A. Lott, K. L. Lear, J. J. Figiel, and K. J. Malloy, "Efficient room-temperature continuous-wave AlGaInP/AlGaAs visible $(670 \mathrm{~nm})$ vertical-cavity surface-emitting laser diodes," IEEE Photon. Technol. Lett., vol. 6, pp. 313-316, 1994.

[4] M. H. Crawford, R. P. Schneider, Jr., K. D. Choquette, K. L. Lear, S. P. Kilcoyne, and J. J. Figiel, "High efficiency AlGalnP-based 660-680 nm vertical-cavity surface emitting lasers," Electron. Lett., vol. 31, pp. 196-197, 1994.

[5] R. P. Schneider, Jr., M. H. Crawford, K. D. Choquette, K. L. Lear, S. P. Kilcoyne, and J. J. Figiel, "Improved AlGaInP-based red $(670-690 \mathrm{~nm})$ surface-emitting lasers with novel C-doped short-cavity epitaxial design," Appl. Phys. Lett., accepted for publication.

[6] R. P. Schneider, Jr., J. A. Lott, K. L. Lear, K. D. Choquette, M. H. Crawford, S. P. Kilcoyne, and J. J. Figiel, "Metalorganic vapor phase epitaxial growth of red and infrared vertical-cavity surfaceemitting laser diodes," J. Cryst. Growth, vol. 145, pp. 838-845, 1994.

[7] K. D. Choquette, R. P. Schneider, Jr., and J. A. Lott, "Lasing characteristics of visible AlGaInP/AlGaAs vertical-cavity lasers," Opt. Lett., vol. 19, pp. 969-971, 1994.

[8] B. Tell, K. F. Brown-Goebeler, R. E. Leibenguth, F. M. Baez, and Y. H. Lee, "Temperature dependence of GaAs-AlGaAs vertical cavity surface emitting lasers," Appl. Phys. Lett., vol. 60, pp. 683-685, 1992.

[9] D. B. Young, J. W. Scott, F. H. Peters, M. G. Peters, M. L. Majewski, B. J. Thibeault, Scott W. Corzine, and L. A. Coldren, "Enhanced performance of offset-gain high-barrier vertical-cavity surface-emitting lasers," IEEE J. Quantum Electron., vol. 29, pp. 2013-2021, 1993. 